Homology, Homotopy and Applications, vol.12(2), 2010, pp.335-351

\title{
RELATIONS BETWEEN SLICES AND QUOTIENTS OF THE ALGEBRAIC COBORDISM SPECTRUM
}

\author{
MARKUS SPITZWECK
}

\author{
(communicated by J. Daniel Christensen)
}

\begin{abstract}
We prove a relative statement about the slices of the algebraic cobordism spectrum. If the map from MGL to a certain quotient of MGL introduced by Hopkins and Morel is the map to the zeroslice then a relative version of Voevodsky's conjecture on the slices of MGL holds true. We outline the picture for $K$-theory and rational slices.
\end{abstract}

\section{Introduction}

In this paper we discuss certain aspects of the slice filtration of the algebraic cobordism spectrum MGL. The slice filtration was introduced in [23]. It is a filtration on any motivic spectrum which can be thought of as an analogue of the Postnikov tower of a topological spectrum. We discuss the relation between the slice conjecture on MGL [23, Conjecture (5)] and quotients of MGL. The conjecture describes the slices in terms of the motivic Eilenberg Mac Lane spectrum and the topological coefficients $M U_{*}$. The quotients are defined using the classifying map $L_{*} \rightarrow M G L_{* *}$ of the formal group law induced by the canonical orientation of MGL and canonical generators $x_{i}$ of $L_{*}$. Here $L_{*}$ denotes the graded Lazard ring. In topology it is well known that the quotient $\mathrm{MU} /\left(x_{1}, x_{2}, \ldots\right) \mathrm{MU}$ is isomorphic to the Eilenberg Mac Lane spectrum on the integers. This follows essentially from Quillen's theorem $\mathrm{L}_{*} \cong \mathrm{MU}_{*}$ and the particular structure of $\mathrm{L}_{*}$. In motivic homotopy theory no direct analog of this argument seems to work. In particular, the filtration on MGL obtained by dividing out the $x_{i}$ (more precisely ideals of $\mathrm{L}_{*}$ consisting of elements of degree greater than a given bound) is not directly reflected on the homotopy groups of MGL, instead this filtration is conjecturally the slice filtration of MGL. We show that if this holds on the zeroth level then it holds in all levels. The statement is of a purely homotopy theoretic nature and could be formulated in any context of highly structured ring spectra.

Over perfect fields the zero slice of the sphere spectrum is known [22], [10]. An effectivity result for MGL implies that MGL has the same zero slice, see Corollary (3.3).

Our main statement implies that if the quotient of MGL by all the $x_{i}$ coincides with this zero slice then [23, Conjecture (5)] holds, see Corollary (4.9). As a consequence,

Received June 16, 2010, revised August 20, 2010; published on November 8, 2010.

2000 Mathematics Subject Classification: 14F42, 55N22, 19E20.

Key words and phrases: slice, algebraic cobordism spectrum, K-theory.

This article is available at http://intlpress.com/HHA/v12/n2/a11

Copyright (C) 2010, International Press. Permission to copy for private use granted. 
under the same assumption, slices of all Landweber exact spectra are given in terms of the Landweber coefficients and the motivic Eilenberg Mac Lane spectrum, see [18].

The author was inspired by the course notes $[\mathbf{9}]$.

Here is an overview of the sections. In the first section we show effectivity and cellularity results for the algebraic cobordism spectrum. Theorem (3.1) states that the cofiber of the unit map of MGL lies in a certain subcategory of the stable motivic homotopy category spanned by positive Tate-spheres. As a corollary we obtain the effectivity of MGL and a proof of the observation in [23] that the zero slices of the sphere spectrum and MGL coincide. These results can be viewed as refinements to the cellularity results of [2]. The effectivity of MGL was implicitly assumed in [18].

The second paragraph deals with our main observation that if the Hopkins-Morel quotient of MGL is the zero slice of MGL then a relative version of Voevodsky's conjecture $[\mathbf{2 3}]$ on slices holds true, Theorem (4.7). This is closely related to the work of Hopkins and Morel on the spectral sequence for MGL in terms of motivic cohomology, see [11]. In particular it is an unpublished result due to Hopkins and Morel that over fields of characteristic 0 the quotient of MGL by the $x_{i}$ is isomorphic to the motivic Eilenberg Mac Lane spectrum, [9]. It thus follows form Corollary (4.9) that [23, Conjecture (5)] holds over fields of characteristic 0 assuming the HopkinsMorel isomorphism. As above we get under the same assumption the structure of the slices of Landweber spectra.

We note that the idea of using filtrations of the ideal of the Lazard ring spanned by elements of positive degree in a homotopy way goes back to [9].

The third paragraph covers the relationship of quotients of MGL to the algebraic $K$-theory spectrum. The main statement is also contained in [9].

In the last paragraph we consider rational slices. Due to the rational splitting of MGL this simplifies to understanding the rational Landweber theory LQ for the additive formal group law over the rationals, see [13]. We obtain these assertions over regular base schemes by comparing the Landweber decomposition of $K G L_{\mathbb{Q}}$ and a decomposition obtained in [15].

\section{Acknowledgements}

I thank the lecturers of [9]. I thank Niko Naumann, Paul Arne Østvær, Ivan Panin and Oliver Röndigs for inspiring communications and Ulrich Bunke and Ansgar Schneider for helpful comments. I also would like to thank the referee for useful suggestions.

\section{Conventions}

Throughout the article we work over a Noetherian base scheme of finite Krull dimension $S$. The stable motivic homotopy category over $S$ is denoted by $\mathbf{S H}(S)$.

The standard spheres are denoted by $S^{p, q} \cong S_{s}^{\wedge(p-q)} \wedge \mathbb{G}_{m}^{\wedge q}$. The category of smooth schemes over $S$ is denoted by $\mathrm{Sm} / S$. The tensor unit of $\mathbf{S H}(S)$, i.e., the sphere spectrum, is denoted by $\mathbf{1}$.

The full subcategory of $\mathbf{S H}(S)$ of effective spectra is denoted by $\mathbf{S H}(S)^{\text {eff }}$. It is the full localizing triangulated subcategory generated by all $\Sigma_{T}^{\infty} X_{+}, X \in \mathrm{Sm} / S$. 
The $i$-th slice of a motivic spectrum $\mathrm{E}$ is denoted $s_{i}(\mathrm{E})$, see $[\mathbf{2 3}]$.

Throughout the text we will use the language of model categories. An injective model structure will be a model structure e.g., on a diagram category where weak equivalences and cofibrations are understood objectwise. Dually the projective model structure has objectwise fibrations. For homotopy colimits and limits we refer to [5]. For symmetric monoidal model categories we refer to [7].

\section{Effectivity of MGL}

Let $\mathbf{S H}(S)_{\mathcal{T}}$ be the full localizing triangulated subcategory of $\mathbf{S H}(S)$ spanned by $\left\{S^{p, q} \mid p, q \in \mathbf{Z}\right\}$, see [13]. We let $\mathbf{S H}(S)_{\mathcal{T}_{\geqslant 0}}$ be the full localizing triangulated subcategory of $\mathbf{S H}(S)$ spanned by $\left\{S^{p, q} \mid p \in \mathbf{Z}, q \geqslant 0\right\}$.

Theorem 3.1. The cofiber of the unit map $\mathbf{1} \rightarrow \mathrm{MGL}$ is contained in $\Sigma_{T} \mathbf{S H}(S)_{\mathcal{T}_{\geqslant 0}}$.

Corollary 3.2. We have $\mathrm{MGL} \in \mathbf{S H}(S)_{\mathcal{T}_{\geqslant 0}}$. In particular $\mathrm{MGL}$ is an effective spectrum.

Proof. Follows from $\mathbf{1} \in \mathbf{S H}(S)_{\mathcal{T}_{\geqslant 0}}$.

Corollary 3.3. The unit map $\mathbf{1} \rightarrow \mathrm{MGL}$ induces an isomorphism

$$
s_{0}(\mathbf{1}) \stackrel{\cong}{\longrightarrow} s_{0}(\mathrm{MGL}) \text {. }
$$

Proof. The functor $s_{0}$ is triangulated and $s_{0}(X)=0$ for any $X \in \Sigma_{T} \mathbf{S H}(S)^{\text {eff }}$.

We start with preparations for the proof of theorem (3.1).

Lemma 3.4. Let $r$ be an integer and let

$$
S^{2 r, r} \rightarrow X \rightarrow Y \rightarrow S^{2 r, r}[1]
$$

and

$$
X \rightarrow Z \rightarrow W \rightarrow X[1]
$$

be two triangles in $\mathbf{S H}(S)$. Suppose $Y, W \in \Sigma_{T}^{r+1} \mathbf{S H}(S)_{\mathcal{T}_{\geqslant 0}}$. Then the cofiber of the map $S^{2 r, r} \rightarrow Z$ is in $\Sigma_{T}^{r+1} \mathbf{S H}(S)_{\mathcal{T}_{\geqslant 0}}$.

Proof. The cofiber of $S^{2 r, r} \rightarrow Z$ is an extension of $W$ by $Y$.

Lemma 3.5. Let $i: Z \rightarrow X$ be a closed immersion in $\mathrm{Sm} / S$ and $\mathcal{E}$ a vector bundle over $X$. Let $U=X \backslash Z$, and denote the restrictions of $\mathcal{E}$ to $Z$ and $U$ by $\mathcal{E}_{Z}$ and $\mathcal{E}_{U}$, respectively. Let $\mathcal{N}$ be the normal bundle of $i$. Then the cofiber of $\operatorname{Th}\left(\mathcal{E}_{U}\right) \rightarrow \operatorname{Th}(\mathcal{E})$ is canonically isomorphic to $\operatorname{Th}\left(\mathcal{E}_{Z} \oplus \mathcal{N}\right)$ in the $\mathbf{A}^{1}$-homotopy category.

Proof. This follows from the fact that this cofiber is (as Zariski sheaves) isomorphic to $\mathcal{E} /(\mathcal{E} \backslash j(Z)), j$ the composition of $i$ followed by the zero section of $\mathcal{E}$, and that the normal bundle of $j$ is $\mathcal{E}_{Z} \oplus \mathcal{N}$. 
We first quote some facts from [13] about finite Grassmannians, see also [4, Ch. 14]. We let $\mathrm{G}(n, d)$ be the scheme parameterizing locally free quotients of rank $d$ of the trivial bundle of rank $n$. There is a universal short exact sequence

$$
0 \longrightarrow \mathcal{K}_{n, d} \longrightarrow \mathcal{O}_{\mathrm{G}(n, d)}^{n} \longrightarrow \mathcal{Q}_{n, d} \longrightarrow 0
$$

of vector bundles on $\mathrm{G}(n, d)$, and letting $\mathcal{K}_{n, d}^{\prime}$ be the dual of $\mathcal{K}_{n, d}$, the tangent bundle is given by

$$
\mathcal{T}_{\mathrm{G}(n, d)} \cong \mathcal{Q}_{n, d} \otimes \mathcal{K}_{n, d}^{\prime}
$$

The map

$$
i: \mathrm{G}(n, d) \longleftrightarrow \mathrm{G}(n+1, d+1)
$$

classifying $\mathcal{K}_{n, d} \subseteq \mathcal{O}_{\mathrm{G}(n, d)}^{n} \hookrightarrow \mathcal{O}_{\mathrm{G}(n, d)}^{n+1}$ is a closed immersion. From (2) it follows that the normal bundle $\mathcal{N}(i)$ of $i$ identifies with $\mathcal{K}_{n, d}^{\prime}$. Next consider the composition on $\mathrm{G}(n+1, d+1)$

$$
\alpha: \mathcal{O}_{\mathrm{G}(n+1, d+1)}^{n} \longrightarrow \mathcal{O}_{\mathrm{G}(n+1, d+1)}^{n+1} \longrightarrow \mathcal{Q}_{n+1, d+1}
$$

for the inclusion into the first $n$ factors. The complement of the support of $\operatorname{coker}(\alpha)$ is an open subscheme $U \subseteq \mathrm{G}(n+1, d+1)$ and there is a map $\pi: U \rightarrow \mathrm{G}(n, d+1)$ classifying $\left.\alpha\right|_{U}$. It is easy to see that $\pi$ identifies with $\mathcal{Q}_{n, d+1} \rightarrow \mathrm{G}(n, d+1)$. An argument with geometric points reveals that $U=\mathrm{G}(n+1, d+1) \backslash i(\mathrm{G}(n, d))$. Moreover, the natural map $\iota: \mathrm{G}(n, d+1) \rightarrow \mathrm{G}(n+1, d+1)$ classifying the subbundle $\mathcal{K}_{n, d+1} \oplus \mathcal{O} \subset$ $\mathcal{O}^{n+1}$ is the zero section $\mathrm{G}(n, d+1) \rightarrow \mathcal{Q}_{n, d+1}$ followed by the inclusion $\mathcal{Q}_{n, d+1} \cong$ $U \rightarrow \mathrm{G}(n+1, d+1)$.

We summarize the above with a diagram:

$$
\mathrm{G}(n, d) \stackrel{i}{\longrightarrow} \mathrm{G}(n+1, d+1) \prec U \underset{\pi}{\longleftrightarrow} \mathrm{G}(n, d+1) .
$$

We note that compositions of the morphisms $\iota$ yield a map $\bar{\iota}$ : pt $\cong \mathrm{G}(d, d) \rightarrow$ $\mathrm{G}(n, d)$ which we consider as the natural pointing of $\mathrm{G}(n, d)$. Note that the unit of MGL is induced via these maps.

Proposition 3.6. Let $\mathcal{E}$ be a vector bundle of rank $r$ over $\mathrm{G}(n, d)$ which is a finite sum of copies of $\mathcal{K}_{n, d}, \mathcal{K}_{n, d}^{\prime}$ and $\mathcal{O}$. Then $\bar{\iota}^{*} \mathcal{E}$ is canonically trivialized. Furthermore the cofiber of the map of suspension spectra of Thom spaces $S^{2 r, r} \rightarrow \Sigma^{\infty} \mathrm{Th}(\mathcal{E})$ lies in $\Sigma_{T}^{r+1} \mathbf{S H}(S)_{\mathcal{T}_{\geqslant 0}}$.

Proof. We prove the statement by induction on $n$. It clearly holds for $n=0$. Suppose $n \geqslant 0$ and assume the statement holds for $n$. The statement holds for $\mathrm{G}(n+1,0)$ and $\mathrm{G}(n+1, n+1)$. Let $0 \leqslant d<n$. We prove the statement for $\mathrm{G}(n+1, d+1)$. Let $\mathcal{E}$ be a vector bundle on $\mathrm{G}(n+1, d+1)$ of the considered type. It is canonically trivialized over the pointing. We consider the diagram (3). By lemma (3.5) we get an induced exact triangle

$$
\Sigma^{\infty} \operatorname{Th}\left(\mathcal{E}_{U}\right) \longrightarrow \Sigma^{\infty} \operatorname{Th}(\mathcal{E}) \longrightarrow \Sigma^{\infty} \operatorname{Th}\left(\mathcal{E}_{\mathrm{G}(n, d)} \oplus \mathcal{K}_{n, d}^{\prime}\right) \longrightarrow \Sigma^{\infty} \operatorname{Th}\left(\mathcal{E}_{U}\right)[1] .
$$

We note $\left.\mathcal{K}_{n+1, d+1}\right|_{\mathrm{G}(n, d)} \cong \mathcal{K}_{n, d}$, hence $\mathcal{E}_{\mathrm{G}(n, d)}$ and $\mathcal{E}_{\mathrm{G}(n, d)} \oplus \mathcal{K}_{n, d}^{\prime}$ are vector bundles on $\mathrm{G}(n, d)$ of the considered type. 
Since $\iota^{*} \mathcal{K}_{n+1, d+1} \cong \mathcal{K}(n, d+1) \oplus \mathcal{O}$, there is an induced map $\operatorname{Th}\left(\mathcal{K}_{n, d+1} \oplus \mathcal{O}\right) \rightarrow$ $\operatorname{Th}\left(\mathcal{K}_{n+1, d+1}\right)$ which factors through $\operatorname{Th}\left(\left.\mathcal{K}_{n+1, d+1}\right|_{U}\right)$. The map $\operatorname{Th}\left(\mathcal{K}_{n, d+1} \oplus \mathcal{O}\right) \rightarrow$ $\operatorname{Th}\left(\left.\mathcal{K}_{n+1, d+1}\right|_{U}\right)$ and more generally $\operatorname{Th}\left(\iota^{*} \mathcal{E}\right) \rightarrow \operatorname{Th}\left(\mathcal{E}_{U}\right)$ are motivic weak equivalences: we cover $U$ by the opens for which $\alpha$ restricted to a fixed subset of the summands of $\mathcal{O}^{n}$ of size $d+1$ surjects onto $\mathcal{Q}_{n+1, d+1}$. Those opens are pullbacks from $\mathrm{G}(n, d+1)$. We claim on such opens the situation trivializes completely. Without loss of generality we can assume the first $d+1$ summands of $\mathcal{O}^{n}$ surject onto $\mathcal{Q}_{n+1, d+1}$. Then $\mathcal{K}_{n+1, d+1}$ restricted to this open trivializes by projecting to the last $n-d$ summands of $\mathcal{O}^{n+1}$. This trivialization is compatible with the one over the corresponding open $V \subset \mathrm{G}(n, d+1)$. Thus $\left(\left.\left(\iota^{*} \mathcal{E}\right)\right|_{V}\right)^{\circ} \rightarrow\left(\left.\mathcal{E}\right|_{\pi^{-1}(V)}\right)^{\circ}$ is a motivic weak equivalence, and the same holds for the map $\left(\iota^{*} \mathcal{E}\right)^{\circ} \rightarrow \mathcal{E}_{U}^{\circ}$ by a Mayer-Vietoris argument. This shows the claim that the map of Thom spaces is also a motivic weak equivalence.

We can thus rewrite the sequence (4) as

$$
\Sigma^{\infty} \operatorname{Th}\left(\iota^{*} \mathcal{E}\right) \longrightarrow \Sigma^{\infty} \operatorname{Th}(\mathcal{E}) \longrightarrow \Sigma^{\infty} \operatorname{Th}\left(\mathcal{E}_{\mathrm{G}(n, d)} \oplus \mathcal{K}_{n, d}^{\prime}\right) \longrightarrow \Sigma^{\infty} \operatorname{Th}\left(\iota^{*} \mathcal{E}\right)[1]
$$

and use the fact that $\iota^{*} \mathcal{E}$ is of the type considered for $\mathrm{G}(n, d+1)$. By induction hypothesis the cofiber of $S^{2 r, r} \cong \Sigma^{\infty} \operatorname{Th}\left(\bar{\iota}^{*} \iota^{*} \mathcal{E}\right) \rightarrow \Sigma^{\infty} \operatorname{Th}\left(\iota^{*} \mathcal{E}\right)$ lies in $\Sigma_{T}^{r+1} \mathbf{S H}(S)_{\mathcal{T}_{\geqslant 0}}$.

Moreover again by induction hypothesis $\Sigma^{\infty} \mathrm{Th}\left(\mathcal{E}_{\mathrm{G}(n, d)} \oplus \mathcal{K}_{n, d}^{\prime}\right) \in \Sigma_{T}^{r+j} \mathbf{S H}(S)_{\mathcal{T}_{\geqslant 0}}$ with $j=n-d>0$. Now the statement follows from lemma (3.4).

Proof of Theorem (3.1). We let $\mathbf{B G L}_{n}=\operatorname{colim}_{d} \mathrm{G}(n+d, d), \xi_{n}=\operatorname{colim}_{d} \mathcal{K}_{n+d, d}$ the universal vector bundle. Then

$$
\mathrm{MGL}=\operatorname{hocolim}_{n} \Sigma^{-2 n,-n} \Sigma^{\infty} \operatorname{Th}\left(\xi_{n}\right) \cong \operatorname{hocolim}_{n, d} \Sigma^{-2 n,-n} \Sigma^{\infty} \operatorname{Th}\left(\mathcal{K}_{n+d, d}\right) .
$$

The unit $\mathbf{1} \rightarrow \mathrm{MGL}$ is induced via the maps

$$
\Sigma^{-2 n,-n} \Sigma^{\infty} \operatorname{Th}\left(\iota^{*} \mathcal{K}_{n+d, d}\right) \rightarrow \Sigma^{-2 n,-n} \Sigma^{\infty} \operatorname{Th}\left(\mathcal{K}_{n+d, d}\right) .
$$

By proposition (3.6) the cofibers of these maps are in $\Sigma_{T} \mathbf{S H}(S)_{\mathcal{T}_{\geqslant 0}}$. Since cofiber sequences are compatible with homotopy colimits the claim follows.

\section{Quotients and slices of MGL}

Let MGL denote a fibrant and cofibrant model as commutative $\mathbb{S}$-algebra of the algebraic cobordism spectrum. We work in the simplicial version of the $\mathbb{S}$-modules of [3], see [16]. In particular MGL is fibrant and cofibrant as (symmetric) $T$-spectrum. We let $\operatorname{Mod}(\mathrm{MGL})$ be the symmetric monoidal category with weak unit of MGLmodules. The homotopy category of $\operatorname{Mod}(\mathrm{MGL})$ is denoted by $\mathcal{D}_{\mathrm{MGL}}$. It is a closed tensor triangulated category.

We denote $\varphi: \mathrm{MU}_{*} \rightarrow \mathrm{MGL}_{*}$ the canonical map (here $\mathrm{MGL}_{i}=\mathrm{MGL}_{2 i, i}$ by definition) and fix an isomorphism $\mathbf{M U}_{*} \cong \mathbf{Z}\left[x_{1}, x_{2}, \ldots\right],\left|x_{i}\right|=i$ (we divide the usual topological grading by 2 ).

As in topology we can form the quotient $\mathrm{Q}:=\mathrm{MGL} /\left(x_{1}, x_{2}, \ldots\right) \mathrm{MGL}$ by taking iterated cofibers of multiplications by the $x_{i}$ in $\mathcal{D}_{\mathrm{MGL}}$. More precisely, if the quotient $\mathrm{MGL} /\left(x_{1}, \ldots, x_{n-1}\right), n \geqslant 1$, is already defined as object of $\mathcal{D}_{\mathrm{MGL}}$ we define the 
MGL-module MGL $/\left(x_{1}, \ldots, x_{n}\right)$ to be the cofiber of the multiplication by $x_{n}$ map on $\mathrm{MGL} /\left(x_{1}, \ldots, x_{n-1}\right)$, i.e., we have an exact triangle

$$
\begin{gathered}
\Sigma^{2 n, n} \mathrm{MGL} /\left(x_{1}, \ldots, x_{n-1}\right) \stackrel{x_{n}}{\longrightarrow} \mathrm{MGL} /\left(x_{1}, \ldots, x_{n-1}\right) \rightarrow \mathrm{MGL} /\left(x_{1}, \ldots, x_{n}\right) \\
\rightarrow \Sigma^{2 n+1, n} \mathrm{MGL} /\left(x_{1}, \ldots, x_{n-1}\right) .
\end{gathered}
$$

The MGL-module $\mathrm{Q}=\mathrm{MGL} /\left(x_{1}, x_{2}, \ldots\right) \mathrm{MGL}$ is then defined to be the homotopy colimit hocolim $\lim _{n \rightarrow \infty} \mathrm{MGL} /\left(x_{1}, \ldots, x_{n}\right)$ in $\mathcal{D}_{\mathrm{MGL}}$.

This quotient is well-defined up to isomorphism.

We give a construction of a more explicit model of this quotient.

We pick once and for all the following data:

1. $S^{2 i, i}=T^{\wedge i}, i>0$, a cofibrant model of the $(2 i, i)$-sphere in $T$-spectra, and denote the corresponding cofibrant spherical MGL-module $\left(S^{2 i, i} \wedge \mathbb{S}\right) \triangleright \mathrm{MGL}$ (for notation see [16, section 9]) by $\Sigma^{2 i, i} \mathrm{MGL}$.

2. a map $\Sigma^{2 i, i} \mathrm{MGL} \rightarrow \mathrm{MGL}$ representing the element $\varphi_{*}\left(x_{i}\right) \in \mathrm{MGL}_{2 i, i}$, also denoted by $x_{i}$. (We note that by adjunction this map corresponds to a map $S^{2 i, i} \rightarrow \mathrm{MGL}$ in symmetric $T$-spectra, where such a representative exists since $S^{2 i, i}$ is cofibrant and MGL underlying fibrant.)

We let $I$ be the following category: the objects are (commutative) monomials in the $x_{i}$, there is a unique map from $M$ to $N$ if $N$ divides $M$; the monomial 1 is allowed. We let $I^{\circ}$ be the full subcategory consisting of all non-constant monomials. The subcategory $I_{\leqslant 1}$ consists of monomials containing each $x_{i}$ with multiplicity at most $1, I_{\leqslant 1}^{\circ}$ is the same category with the constant monomial removed.

We let $\underline{1}$ be the category whose diagrams are morphisms, i.e., two objects 0 and 1 and one non-identity map. We let $\underline{1}^{n} \subset I_{\leqslant 1}$ be the inclusion of the monomials containing only the $x_{i}, i \leqslant n$. Via these inclusions $I_{\leqslant 1}$ is the union of all the $\underline{1}^{n}$.

We are going to define the following $I$-diagram of MGL-modules: a monomial $x_{1}^{k_{1}} \cdots x_{n}^{k_{n}}, k_{i} \geqslant 0$, is sent to $\left(\Sigma^{2,1} \mathrm{MGL}\right)^{\wedge_{\mathrm{MGL}} k_{1}} \wedge_{\mathrm{MGL}} \cdots \wedge_{\mathrm{MGL}}\left(\Sigma^{2 n, n} \mathrm{MGL}\right)^{\wedge_{\mathrm{MGL}} k_{n}}$. Here by convention we leave out a factor completely if some $k_{i}=0$ as opposed to the fact that we could have included a factor MGL for the empty tensor product (note these two possibilities indeed differ since MGL only figures as a weak unit). Also by convention the monomial 1 is sent to MGL. The morphisms will be given by multiplications with the $x_{i}$, i.e., iterations of the morphisms $x_{i}$. We have to be careful since in general the two possible maps $\Sigma^{2 i, i} \mathrm{MGL} \wedge \mathrm{MGL} \Sigma^{2 i, i} \mathrm{MGL} \rightarrow \Sigma^{2 i, i} \mathrm{MGL}$ given by applying the map $x_{i}$ either on the left or on the right and then composing with a unit morphism (note MGL only serves as a weak unit) do not coincide in general. Therefore we make the convention that for a map $M \rightarrow N$ in $I, M=x_{1}^{k_{1}} \cdots x_{n}^{k_{n}}, N=x_{1}^{l_{1}} \cdots x_{n}^{l_{n}}, l_{i} \leqslant k_{i}$, we insert for any $1 \leqslant i \leqslant n$ the map $\left(\Sigma^{2 i, i} \mathrm{MGL}\right)^{\wedge_{\mathrm{MGL}} k_{1}} \rightarrow\left(\Sigma^{2 i, i} \mathrm{MGL}\right)^{\wedge_{\mathrm{MGL}} l_{1}}$ which applies $x_{i}$ on the $k_{i}-l_{i}$ rightmost tensor factors of the source followed by appropriate unit maps. The order in which these unit maps are applied and to which tensor factors they are applied does not matter by the properties of a symmetric monoidal model category with weak unit.

We end up with a diagram of MGL-modules, denoted D.

Lemma 4.1. The diagram $\mathrm{D}$ is commutative. 
Proof. As already remarked the transition maps in D are well-defined. The commutativity of $\mathrm{D}$ follows now again from the properties of a symmetric monoidal model category with weak unit, more precisely from the fact that for maps $f$ and $g$ between MGL-modules we have $\left(f \wedge_{M G L}\right.$ id $) \circ\left(\right.$ id $\left.\wedge_{M G L} g\right)=f \wedge_{M G L} g$.

We need the following lemma in which we denote by hofib the homotopy fiber of a map between pointed simplicial sets.

Lemma 4.2. Let

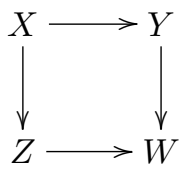

be a commutative diagram of pointed simplicial sets. Let $P$ be the homotopy pullback of the right lower triangle of the square. Then the homotopy fiber of the natural map $X \rightarrow P$ is naturally equivalent to hofib(hofib $(X \rightarrow Z) \rightarrow \operatorname{hofib}(Y \rightarrow W)$ ).

Proof. Replacing the diagram with an injectively fibrant diagram the statement follows from the corresponding strict statement.

Let $\mathrm{D}_{\leqslant 1}$ be the restriction of $\mathrm{D}$ to $I_{\leqslant 1}$ and $\mathrm{D}_{\leqslant 1}^{\circ}$ the one to $I_{\leqslant 1}^{\circ}$.

We have the following observations. We use the notion of total cofiber of a diagram with respect to a subdiagram, see [8]. This is defined to be the cofiber of the natural map from the homotopy colimit of the subdiagram to the homotopy colimit of the total diagram. Usually this can be viewed as the total object corresponding to a diagram of a certain shape, e.g., of a cubical diagram. For a functor to be homotopy right cofinal see [5, Definition 19.6.1].

Lemma 4.3. 1. The inclusion $I_{\leqslant 1}^{\circ} \rightarrow I^{\circ}$ is homotopy right cofinal.

2. The total cofiber of the diagram $\mathrm{D}_{\leqslant 1}$ with respect to the inclusion $\mathrm{D}_{\leqslant 1}^{\circ} \rightarrow \mathrm{D}_{\leqslant 1}$, i.e., the cofiber of the map hocolim $\mathrm{D}_{\leqslant 1}^{\circ} \rightarrow \mathrm{D}(1)=\mathrm{MGL}$, is isomorphic to $\mathrm{Q}$ in $\mathcal{D}_{\mathrm{MGL}}$.

Proof. (1): Let the inclusion be denoted by $j$. We have to show that for any object $o \in I^{\circ}$ the under category $o \backslash j$ is contractible. But this under category has the initial object $\left(o^{\prime}, o \rightarrow o^{\prime}\right)$, where $o^{\prime}$ contains $x_{i}$ with multiplicity 1 if $x_{i} \mid o$, otherwise it contains it with multiplicity 0.

(2): We first show the analogous statement for finitely many $x_{i}$ : the total cofiber of $\left.\mathrm{D}\right|_{\underline{1}^{n}}$ with respect to the inclusion $\left(\underline{1}^{n}\right)^{\circ} \subset \underline{1}^{n}$ is equivalent to $\mathrm{MGL} /\left(x_{1}, \ldots, x_{n}\right) \mathrm{MGL}$. This is proved by induction on $n$ using the following statement (with $J=\underline{1}^{n}$ for the induction step $n \mapsto n+1)$ :

Let $J^{\circ}$ be a small category and let $J$ be the same category added a terminal object (so if $J$ already had a terminal object this object will no longer be terminal). Let $\mathcal{C}$ be a pointed model category and $G: J \times \underline{1} \rightarrow \mathcal{C}$ be a functor. Let $(J \times \underline{1})^{\circ}$ be again the category obtained by removing the terminal object. Then the total cofiber of $G$ with respect to the inclusion $(J \times \underline{1})^{\circ} \rightarrow J \times \underline{1}$ can be computed as follows: it is the cofiber of the map $\operatorname{Totcof}\left(\left.G\right|_{J \times\{0\}}\right) \rightarrow \operatorname{Totcof}\left(\left.G\right|_{J \times\{1\}}\right)$, Totcof denoting the 
total cofiber with respect to the inclusions of the respective subcategories obtained by removing the terminal object.

By considering pointed mapping spaces this statement reduces to the dual statement for pointed simplicial sets. Here to compute homotopy limits we can use the injective model structure on diagram categories. We let $K$ be the opposite of $J$, similarly with $K^{\circ}$. The category $(K \times \underline{1})^{\circ}$ this time denotes $K \times \underline{1}$ with the initial object removed. Let $G: K \times \underline{1} \rightarrow$ sSet. be a functor. Fix an injectively fibrant replacement $G \rightarrow R$. We note that $\left.R\right|_{(K \times \underline{1})^{\circ}},\left.R\right|_{K^{\circ} \times\{i\}}, i=0,1$, are again injectively fibrant: the respective restriction functors are right Quillen functors since the corresponding adjoints preserve objectwise cofibrations.

Let $\mathrm{i}$ be the initial object of $K$. We replace the diagram $R$ by the square

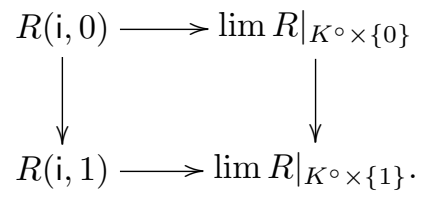

This yields again an injectively fibrant diagram, the limit of the lower right triangle

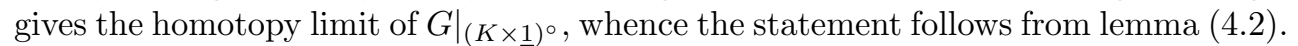

We are left to prove the statement for infinitely many $x_{i}$. The restriction functors from $I_{\leqslant 1}^{\circ}$-diagrams to $\left(\underline{1}^{n}\right)^{\circ}$-diagrams preserve projectively cofibrant diagrams,

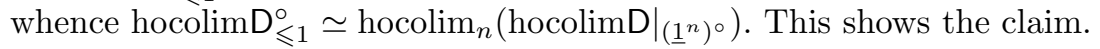

For any $n>0$ let $I_{\mathrm{deg} \geqslant n}$ be the subcategory of $I$ of monomials of degree $\geqslant n$, where the degree of a monomial $x_{1}^{k_{1}} \cdots x_{n}^{k_{n}}$ is $\sum_{i=1}^{n} i \cdot k_{i}$. Moreover for a monomial $M$ let $I_{>M}$ be the subcategory of all monomials which are divisible by $M$, except $M$ itself. We also let $\mathrm{D}_{\mathrm{deg} \geqslant n}=\left.\mathrm{D}\right|_{I_{\mathrm{deg} \geqslant n}}$.

Proposition 4.4. Let $F: I_{\mathrm{deg} \geqslant n} \rightarrow \mathcal{C}$ be a diagram in a cofibrantly generated model category $\mathcal{C}$ such that for any monomial $M$ of degree $n$ the natural map

$$
\left.\operatorname{hocolim} F\right|_{I_{>M}} \rightarrow F(M)
$$

is an equivalence. Then the natural map

$$
\operatorname{hocolim}\left(\left.F\right|_{I_{\mathrm{deg} \geqslant n+1}}\right) \rightarrow \operatorname{hocolim} F
$$

is an equivalence.

Proof. Let $Q \rightarrow F$ be a cofibrant replacement of $F$ for the projective model structure on $\mathcal{C}^{I_{\mathrm{deg} \geqslant n}}$. Let $M$ be a monomial of degree $n$. We claim $\left.Q\right|_{I_{>M}}$ is still cofibrant: in fact the right adjoint $r$ to the restriction functor preserves objectwise fibrations: for $o \in I_{>M}$ we have $r(H)(o)=H(o)$, for $o \notin I_{>M}$ we have $r(H)(o)=$ pt.

Thus if we define $Q^{\prime}$ by replacing for any $M$ with $\operatorname{deg}(M)=n$ the object $Q(M)$ with colim $\left.Q\right|_{I_{>M}}$ and leaving the other entries unchanged we do not change the weak homotopy type of $Q$. As above $\left.Q^{\prime}\right|_{I_{\mathrm{deg} \geqslant n+1}}$ is cofibrant, moreover $Q^{\prime}$ is cofibrant itself since for any $B \in \mathcal{C}^{I_{\mathrm{deg} \geqslant n}}$ we have $\operatorname{Hom}\left(\left.Q^{\prime}\right|_{I_{\mathrm{deg} \geqslant n+1}},\left.B\right|_{I_{\mathrm{deg} \geqslant n+1}}\right) \cong \operatorname{Hom}\left(Q^{\prime}, B\right)$. Thus

$$
\left.\left.\operatorname{hocolim} Q^{\prime}\right|_{I_{\mathrm{deg} \geqslant n+1}} \simeq \operatorname{colim} Q^{\prime}\right|_{I_{\mathrm{deg} \geqslant n+1}} \cong \operatorname{colim} Q^{\prime} \simeq \operatorname{hocolim} Q^{\prime} \simeq \operatorname{hocolim} Q,
$$

which finishes the proof. 
For the proof of the next statement we use the notion of a left Quillen presheaf on $\omega$, where $\omega$ is the first infinite ordinal. In this case such a presheaf is given by a model category $\mathcal{C}_{n}$ for each natural number $n$ and left Quillen functors $f_{n}: \mathcal{C}_{n+1} \rightarrow \mathcal{C}_{n}$ for each $n \geqslant 0$. A section consists of objects $X_{n} \in \mathcal{C}_{n}$ for each $n \geqslant 0$ and maps $f_{n}\left(X_{n+1}\right) \rightarrow X_{n}, n \geqslant 0$. It is called homotopy cartesian if the maps $\left(\mathbf{L} f_{n}\right)\left(X_{n+1}\right) \rightarrow X_{n}$ are isomorphisms in $\mathrm{Ho}\left(\mathcal{C}_{n}\right)$. The category of sections possesses the injective model structure where weak equivalences and cofibrations are objectwise. The proof is analogous to [7, Theorem 5.1.3]. We will use the fact that the mapping space out of a homotopy cartesian section $X_{\bullet}$ into any $Y_{\bullet}$ is given as the homotopy limit over the individual mapping spaces $\operatorname{map}\left(X_{n}, Y_{n}\right)$, see $[\mathbf{1 9}$, Lemma 3.1, Remark 3.2].

Lemma 4.5. Let $F: \mathcal{C} \leftrightarrow \mathcal{D}: G$ be a Quillen adjunction between stable pointed model categories. Suppose $\mathbf{R} G: \mathrm{HoD} \rightarrow \mathrm{HoC}$ preserves arbitrary sums. Then $\mathbf{R} G$ preserves homotopy colimits.

Proof. We provide a proof. First note that a homotopy colimit of a functor $F: I \rightarrow$ $\mathcal{D}$, which we suppose to take values in cofibrant objects, can be computed as the homotopy colimit of the simplicial diagram $[n] \mapsto \bigsqcup_{\varphi:[n] \rightarrow I} F(\varphi(0))$, where $[n]$ is the ordered set $\{0, \ldots, n\}$ viewed as a category. By considering mapping spaces this reduces to the statement that mapping spaces in $\mathbf{S S e t}{ }^{I}$ can be computed by homotopy ends.

Since $\mathbf{R} G$ preserves homotopy coproducts by assumption it thus suffices to show that colimits over $\triangle^{\mathrm{op}}$ are preserved. Let $\alpha: \triangle^{\mathrm{op}} \rightarrow \mathcal{D}$ be a functor. We claim

$$
\operatorname{hocolim}(\alpha) \simeq \operatorname{hocolim}_{n} \operatorname{hocolim}\left(\left.\alpha\right|_{\triangle_{\leqslant n}^{\mathrm{op}}}\right) .
$$

This proves the above since $\mathbf{R} G$ preserves finite homotopy colimits since we are dealing with a stable situation and sequential homotopy colimits over $\omega$ since $\mathbf{R} G$ preserves sums. Using mapping spaces we reduce the statement about $\alpha$ to the dual statement in simplicial sets. Now we observe that $\left.\mathrm{Ho}_{(\mathbf{s S e t}}{ }^{\triangle}\right)$ is equivalent to homotopy cartesian sections in the homotopy category of the category of sections of the left Quillen presheaf on $\omega, n \mapsto \mathbf{S S e t}^{\triangle \leqslant n}$, the categories $\mathbf{s S e t}^{\triangle \leqslant n}$ carrying the injective model structure such that the restriction maps preserve cofibrations. Considering mapping spaces out of constant diagrams shows the claim since a mapping space between homotopy cartesian sections is a homotopy limit over $\omega$ of the individual mapping spaces.

We now turn to the functors

$$
f_{i}: \mathbf{S H}(S) \rightarrow \Sigma_{T}^{i} \mathbf{S H}(S)^{e f f} \subset \mathbf{S H}(S)
$$

introduced in [23]. These are defined as right adjoints to the inclusions $\Sigma_{T}^{i} \mathbf{S H}(S)^{\text {eff }} \subset$ $\mathbf{S H}(S)$. These functors can be defined on the level of model categories by using colocalization of model categories, see [14]. In particular it makes sense to ask whether these functors preserve homotopy colimits.

Corollary 4.6. The functors $f_{i}$ preserve homotopy colimits.

Proof. This follows from lemma (4.5) and the fact that the $f_{i}$ preserve sums [23]. 
We now can state the main observation of this text. Here for a spectrum $E$ and a free abelian group $A$ we denote by $E \otimes A$ the spectrum which corepresents the functor $X \mapsto \operatorname{Hom}_{\mathbf{A b}}(A, \operatorname{Hom}(E, X))$.

Theorem 4.7. Suppose the natural map $\mathrm{MGL} \rightarrow \mathrm{Q}$ is the map from MGL to its zeroslice. Then [23, Conjecture (5)] holds with $H_{\mathrm{MU}_{2 q}}$ replaced by $s_{0} \mathrm{MGL} \otimes \mathrm{MU}_{2 q}$, i.e., there is a natural isomorphism $s_{q}(\mathrm{MGL}) \cong \Sigma_{T}^{q} s_{0} \mathrm{MGL} \otimes \mathrm{MU}_{2 q}$ compatible with the natural homomorphism $\mathrm{MU}_{*} \rightarrow \mathrm{MGL}_{*}$.

Note that the compatibility with the natural homomorphism $\mathrm{MU}_{*} \rightarrow \mathrm{MGL}_{* *}$ as explained in $[\mathbf{2 3}]$ still makes sense.

Proof. Lemma (4.3) and [5, Theorem 19.6.13] imply that the map from MGL to the total cofiber of $D$ with respect to $D^{\circ} \rightarrow D$ is the map to $Q$, hence by assumption this is the map to the zero-slice. Thus hocolimD ${ }^{\circ} \rightarrow f_{1} \mathrm{MGL}$ is an equivalence. By corollary (4.6) the $f_{i}$ commute with homotopy colimits. We denote by $\mathcal{F}_{i}$ functorial versions of the $f_{i}$ on the level of model categories. Then we can rewrite $f_{2}$ hocolimD ${ }^{\circ} \simeq$ hocolim $\mathcal{F}_{2} \mathrm{D}^{\circ}$. Observe the diagram $\mathcal{F}_{2} \mathrm{D}^{\circ}$ satisfies the assumptions of proposition (4.4) for $n=1$, hence $f_{2} \mathrm{MGL} \simeq$ hocolim $\mathcal{F}_{2} \mathrm{D}^{\circ} \simeq$ hocolim $\mathrm{D}_{\mathrm{deg} \geqslant 2}$.

By increasing the degree of the monomials it follows from proposition (4.4) by induction that $f_{n} \mathrm{MGL} \simeq$ hocolimD $\mathrm{deg}_{2 n}$. In addition the maps

$$
f_{n+1} \mathrm{MGL} \rightarrow f_{n} \mathrm{MGL}
$$

are the naturally induced maps

$$
\text { hocolimD } D_{\operatorname{deg} \geqslant n+1} \rightarrow \text { hocolimD } D_{\operatorname{deg} \geqslant n}
$$

Thus the $s_{n}$ MGL are the cofibers of these maps. We rewrite the source again as hocolim $\mathcal{F}_{n+1} \mathrm{D}_{\mathrm{deg} \geqslant n}$. Cofibers commute with homotopy colimits, thus

$$
s_{n} \mathrm{MGL} \simeq \operatorname{hocolim} \operatorname{cofib}\left(\mathcal{F}_{n+1} \mathrm{D}_{\mathrm{deg} \geqslant n} \rightarrow \mathrm{D}_{\mathrm{deg} \geqslant n}\right) .
$$

The value of this cofiber of diagrams at a monomial of degree $>n$ is zero, at a monomial of degree $n$ we have exactly $\Sigma_{T}^{n} s_{0} \mathrm{MGL}$. Now it is easy to see that the homotopy colimit of such a diagram is the homotopy coproduct of the entries in degree $n$, which is $\Sigma_{T}^{n} s_{0} \mathrm{MGL} \otimes \mathrm{MU}_{2 n}$.

The compatibility with the natural homomorphism $\mathrm{MU}_{*} \rightarrow \mathrm{MGL}_{* *}$ follows by the choice of the $x_{i}$.

Remark 4.8. Since the $s_{n} \mathrm{MGL}$ are the cofibers of the maps (6) which have naturally the structure of maps in $\mathcal{D}_{\mathrm{MGL}}$ it follows that the $s_{n} \mathrm{MGL}$ also have naturally the structure of MGL-modules, i.e., lie in $\mathcal{D}_{\mathrm{MGL}}$. It is natural to ask (as was done by the referee) if the factorization $s_{n} \mathrm{MGL} \cong \Sigma_{T}^{n} s_{0} \mathrm{MGL} \otimes \mathrm{MU}_{2 n}$ can be achieved in $\mathcal{D}_{\mathrm{MGL}}$. Indeed, an alternative to the second step in the above proof of the Theorem shows this is possible: we can form the (homotopy) left Kan extension of $D_{\operatorname{deg} \geqslant n+1}$ along the inclusion $I_{\mathrm{deg} \geqslant n+1} \rightarrow I_{\mathrm{deg} \geqslant n}$ (in MGL-modules). By the above reasoning this is equivalent to $\mathcal{F}_{n+1} \mathrm{D}_{\mathrm{deg} \geqslant n}$ (after forgetting the MGL-module structure). Thus the second step of the above argument goes through in MGL-modules, showing the claim. 
Alternatively we can define a filtration on MGL-modules analogous to the filtration on spectra given by the $f_{i}$ by defining effective MGL-modules as those modules which are generated by the free MGL-modules on effective spectra. The equation $f_{n} \mathrm{MGL} \simeq$ hocolimD $D_{\mathrm{deg} \geqslant n}$ and some adjointness argument shows that the induced filtration on MGL is compatible with our original filtration after forgetting the module structure. Thus going through the above argument entirely in MGL-modules also shows the claim about the decomposition $s_{n} \mathrm{MGL} \cong \Sigma_{T}^{n} s_{0} \mathrm{MGL} \otimes \mathrm{MU}_{2 n}$ as $\mathrm{MGL}-$ modules.

Recall the natural orientation $\mathrm{MGL} \rightarrow \mathrm{MZ}$ of the motivic Eilenberg Mac Lane spectrum. It is additive, in particular the $x_{i}$ all map to zero in $\mathbf{M} \mathbf{Z}_{* *}$. Iteratively we get factorizations $\mathrm{MGL} /\left(x_{1}, \ldots, x_{n}\right) \mathrm{MGL} \rightarrow \mathrm{MZ}$ in $\mathbf{S H}(S)$ in a compatible way, which gives a map $\mathrm{Q} \rightarrow \mathrm{MZ}$.

Corollary 4.9. Suppose $S$ is the spectrum of a perfect field. If the natural map $\mathrm{Q} \rightarrow \mathrm{MZ}$ is an isomorphism then [23, Conjecture (5)] holds.

Proof. By [10] and $[\mathbf{2 2}] s_{0} \mathbf{1} \cong \mathrm{MZ}$. Thus by corollary (3.3) the map MGL $\rightarrow \mathrm{MZ}$ is the map from MGL to its zero-slice. The statement follows from theorem (4.7).

\section{K-theory}

By the Landweber exactness theorem [13] and [12] the spectrum KGL is the Landweber spectrum associated to the $\mathrm{MU}_{*}$-algebra

$$
x_{1}^{-1} \mathrm{MU}_{*} /\left(x_{2}, x_{3}, \ldots\right) \mathrm{MU}_{*} \cong \mathbf{Z}\left[u, u^{-1}\right] .
$$

The latter algebra classifies the multiplicative formal group law $x+y-u x y$.

The orientation MGL $\rightarrow \mathrm{KGL}$ sends all $x_{i} \in \mathrm{MGL}_{2 i, i}, i \geqslant 2$, to $0 \in \mathrm{KGL}_{2 i, i}$. Thus we obtain a factorization $\mathrm{MGL} /\left(x_{2}, x_{3} \ldots\right) \mathrm{MGL} \rightarrow \mathrm{KGL}$. Since $x_{1}$ acts invertibly on KGL this map further factors as

$$
B: x_{1}^{-1} \mathrm{MGL} /\left(x_{2}, x_{3} \ldots\right) \mathrm{MGL} \rightarrow \mathrm{KGL} .
$$

Here for a highly structured ring spectrum $E$, a (highly structured) $E$-module $M$ and an element $a \in E_{2 n, n}$ we set

$$
a^{-1} M=\operatorname{hocolim}\left(M \rightarrow \Sigma^{-2 n,-n} M \rightarrow \Sigma^{-4 n,-2 n} M \rightarrow \cdots\right),
$$

where the transition maps are given by multiplication by $a$.

Lemma 5.1. Let

$$
0 \rightarrow M_{*} \stackrel{f}{\rightarrow} N_{*} \rightarrow Q_{*} \rightarrow 0
$$

be a short exact sequence of evenly graded Landweber exact $\mathrm{MU}_{*}$-modules. Let

$$
F: \mathrm{E}_{M} \rightarrow \mathrm{E}_{N}
$$

be a map of spectra in $\mathbf{S H}(\mathbf{Z})_{\mathcal{T}}$ representing the homology transformation given by $f$ via the motivic Landweber exact functor theorem. Then the cofiber of $F$ represents the Landweber theory given by $Q_{*}$. 
Proof. We let $\mathcal{X}$ be the stack $\left[\operatorname{Spec}\left(\mathrm{MU}_{*}\right) / \operatorname{Spec}\left(\mathrm{MU}_{*} \mathrm{MU}\right)\right]$ and $\widetilde{M}_{*}$, etc., the quasicoherent sheaves on $\mathcal{X}$ obtained from the $M_{*}$, etc., by pushforward along the map $\operatorname{Spec}\left(\mathrm{MU}_{*}\right) \rightarrow \mathcal{X}$. Then by Landweber exactness the sequence

$$
0 \rightarrow \widetilde{M}_{*} \rightarrow \widetilde{N}_{*} \rightarrow \widetilde{Q}_{*} \rightarrow 0
$$

is a short exact sequence of flat $\mathcal{O}_{\mathcal{X}}$-modules. In particular tensoring this sequence with a quasi-coherent $\mathcal{O}_{\mathcal{X}}$-module yields again a short exact sequence. However, the Landweber theorem $([\mathbf{1 3}])$ is proved by considering the MGL-homology of a motivic spectrum as a $\left(\mathrm{MGL}_{*}, \mathrm{MGL}_{*} \mathrm{MGL}\right)$-comodule and via restriction along a map of Hopf algebroids as $\left(\mathrm{MU}_{*}, \mathrm{MU}_{*} \mathrm{MU}\right)$-comodule, then tensoring this over $\mathcal{O}_{\mathcal{X}}$ with $\widetilde{M}_{*}$ and finally pulling back to $\operatorname{Spec}\left(\mathrm{MU}_{*}\right)$. This shows that the sequence of motivic homology theories obtained from the original sequence is short exact. In particular if the first map is represented by any map of motivic spectra then the cofiber will represent the homology theory associated with $Q_{*}$.

Theorem 5.2. The map of spectra $B: x_{1}^{-1} \mathrm{MGL} /\left(x_{2}, x_{3}, \ldots\right) \mathrm{MGL} \rightarrow \mathrm{KGL}$ is an isomorphism.

Proof. We use that $x_{1}^{-1} \mathrm{MGL} /\left(x_{2}, x_{3}, \ldots\right) \mathrm{MGL}$ can also be constructed by first inverting $x_{1}$ and then quotienting out the $x_{i}, i \geqslant 2$. Now observe that all the quotients $x_{1}^{-1} \mathrm{MU}_{*} /\left(x_{2}, \ldots, x_{n}\right) \mathrm{MU}_{*}$ are Landweber exact: they are torsionfree, and for a prime $p$ the element $v_{1}$ of $\left(\mathrm{MU}_{*}\right)_{(p)}$ already acts invertibly. Thus the

$$
\begin{gathered}
0 \rightarrow \Sigma^{n+1} x_{1}^{-1} \mathrm{MU}_{*} /\left(x_{2}, \ldots, x_{n}\right) \mathrm{MU}_{*} \rightarrow x_{1}^{-1} \mathrm{MU}_{*} /\left(x_{2}, \ldots, x_{n}\right) \mathrm{MU}_{*} \\
\rightarrow x_{1}^{-1} \mathrm{MU}_{*} /\left(x_{2}, \ldots, x_{n+1}\right) \mathrm{MU}_{*} \rightarrow 0
\end{gathered}
$$

( $\Sigma$ refers to a shift of evenly graded groups) are short exact sequences of evenly graded Landweber modules, and lemma (5.1) applies. Thus it follows inductively that the quotients ${ }_{1}^{-1} \mathrm{MGL} /\left(x_{2}, \ldots, x_{n}\right) \mathrm{MGL}$ are the Landweber spectra for the modules $x_{1}^{-1} \mathrm{MU}_{*} /\left(x_{2}, \ldots, x_{n}\right) \mathrm{MU}_{*}$. Passing to the colimit shows the claim.

Remark 5.3. Fix a prime $p$ and let $\mathrm{BP}^{\text {Top }}$ be the topological Brown-Peterson spectrum. Let BP be the motivic spectrum on the Landweber coefficients

$$
\mathrm{BP}_{*}^{\text {Top }}=\mathbf{Z}_{(p)}\left[v_{1}, v_{2}, v_{3}, \ldots\right]
$$

$\left|v_{i}\right|=2\left(p^{i}-1\right), i \geqslant 1$. It can be seen that this coincides with the definition given in $[21]$ since both definitions give rise to the universal oriented ring cohomology theory on compact objects with $p$-typical formal group law.

Let $\mathrm{E}(n)^{\text {Top }}$ be the topological spectrum for the coefficients

$$
v_{n}^{-1} \mathrm{BP}_{*}^{\text {Top }} /\left(v_{n+1}, v_{n+2}, \ldots\right) \text {. }
$$

The corresponding motivic Landweber spectrum is denoted $\mathrm{E}(n)$. Now the quotients $v_{n}^{-1} \mathrm{BP}_{*}^{\text {Top }} /\left(v_{n+1}, \ldots, v_{n+k}\right) \mathrm{BP}_{*}^{\text {Top }}$ are also Landweber exact. Hence by the same method as for theorem (5.2) we see that there is an isomorphism

$$
\mathrm{E}(n) \cong v_{n}^{-1} \mathrm{BP} /\left(v_{n+1}, v_{n+2}, \ldots\right) \mathrm{BP},
$$

compare this with [6]. (For forming this quotient and localization use that BP can be obtained as a highly structured $\mathrm{MGL}_{(p)}$-module.) 
The map from [18, par. 6] is ill-defined since BP is not a direct summand of $\mathrm{MGL}_{(p)}$ as an MGL-module.

Next we analyze the relationship to connective or effective $K$-theory. A version of that has been introduced in [10].

Since MGL $/\left(x_{2}, \ldots\right) M G L$ is effective, the canonical map

$$
\mathrm{MGL} /\left(x_{2}, \ldots\right) \mathrm{MGL} \rightarrow x_{1}^{-1} \mathrm{MGL} /\left(x_{2}, \ldots\right) \mathrm{MGL} \cong \mathrm{KGL}
$$

factors as

$$
B^{\text {eff }}: \mathrm{MGL} /\left(x_{2}, \ldots\right) \mathrm{MGL} \rightarrow f_{0} \mathrm{KGL} .
$$

Proposition 5.4. Suppose the map MGL $\rightarrow \mathrm{Q}$ is the map from MGL to its zero-slice. Then $B^{\text {eff }}$ induces isomorphisms on slices. If $S$ is the spectrum of a perfect field and $B^{\text {eff }}$ is an isomorphism, then the map $\mathrm{MGL} \rightarrow \mathrm{Q}$ is the map from MGL to its zero-slice.

Proof. Let us assume MGL $\rightarrow$ Q is the map to the zero-slice. Then by theorem (4.7) the assumption (SlMGL) of [18] is fulfilled, hence by [18, corollary 4.2] we have $s_{0} \mathrm{KGL} \cong s_{0} \mathrm{MGL}$, and the isomorphism is realized by the map $s_{0} \mathrm{MGL} \rightarrow s_{0} f_{0} \mathrm{KGL}$.

By the periodicity of $\mathrm{KGL}$ the map $f_{1} \mathrm{KGL} \rightarrow f_{0} \mathrm{KGL}$ is isomorphic to the map $\Sigma_{T} f_{0} \mathrm{KGL} \rightarrow f_{0} \mathrm{KGL}$ given by multiplication by the Bott element $u \in\left(f_{0} \mathrm{KGL}\right)_{2,1}=$ $\mathrm{KGL}_{2,1}$. Thus the cofiber of this map is isomorphic to $s_{0} \mathrm{MGL}$, and the same holds true for the cofiber of the map $\Sigma_{T} \mathrm{MGL} /\left(x_{2}, \ldots\right) \mathrm{MGL} \rightarrow \mathrm{MGL} /\left(x_{2}, \ldots\right) \mathrm{MGL}$ given by multiplication by $x_{1}$ by the assumption. Iterating this process shows the claim.

Suppose now that $S$ is the spectrum of a perfect field. Then by [10] there are isomorphisms $s_{0} \mathbf{1} \cong s_{0} \mathrm{KGL} \cong \mathrm{MZ}$, and by corollary (3.3) we also have $s_{0} \mathrm{MGL} \cong s_{0} \mathrm{KGL}$. Now if $B^{\text {eff }}$ is an isomorphism, then the map

$$
\Sigma_{T} \mathrm{MGL} /\left(x_{2}, \ldots\right) \mathrm{MGL} \rightarrow \mathrm{MGL} /\left(x_{2}, \ldots\right) \mathrm{MGL}
$$

given by multiplication by $x_{1}$ is the map

$$
f_{1} \mathrm{MGL} /\left(x_{2}, \ldots\right) \mathrm{MGL} \rightarrow \mathrm{MGL} /\left(x_{2}, \ldots\right) \mathrm{MGL}
$$

showing the claim.

\section{Rational slices}

We show in this paragraph that the assumptions from the last sections hold true after rationalization, at least over regular base schemes.

We denote by $L \mathbf{Q}$ the Landweber spectrum associated with the $\mathbf{M U}_{*}$-module $\mathbb{Q}$ carrying the additive formal group law. It is known (see [1, Corollary 15.1.6]) that over geometrically unibranched schemes $\mathbf{L Q}$ is canonically isomorphic to the motivic Eilenberg-Mac Lane spectrum.

We note that any rational motivic Landweber spectrum has a decomposition into a sum of $\Sigma^{2 i, i} \mathbf{L Q}$ for various $i$. This follows directly from the corresponding decomposition of the topological coefficients. 
Since the Landweber coefficients for the rational $K$-theory spectrum are $\mathbb{Q}\left[v, v^{-1}\right]$ ( $v$ is the Bott element in homological degree 2) we obtain

$$
\mathrm{KGL}_{\mathbb{Q}} \cong \mathrm{PLQ}:=\bigoplus_{i \in \mathbf{Z}} \Sigma^{2 i, i} \mathrm{~L} \mathbf{Q}
$$

which we compare also with [13, Theorem 10.1].

Using projectors [15, Theorem IV.72] gives a decomposition

$$
\mathrm{KGL}_{\mathbb{Q}} \cong \bigoplus_{i \in \mathbf{Z}} \mathbb{H}_{\mathrm{L}}^{(i)}
$$

This decomposition is first defined for regular base schemes $S$. For morphisms between regular base schemes it pulls back. It thus makes sense to pull back the decomposition given over $\operatorname{Spec}(\mathbf{Z})$ to any base scheme, which we shall assume.

The next statement follows from the structure of a rational Snaith map

$$
\operatorname{colim}_{n}\left(\mathrm{~L} \mathbf{Q} \wedge \Sigma^{-2 n,-n} \Sigma^{\infty} \mathbf{P}_{+}^{\infty}\right) \rightarrow \mathrm{KGL}_{\mathbb{Q}}
$$

(see [17] for the Snaith map) and the way the projectors for (8) are defined.

We call the map

$$
\Sigma^{-2 i,-i} \Sigma^{\infty} \mathbf{P}_{+}^{\infty} \rightarrow \mathrm{KGL}_{\mathbb{Q}}
$$

the $i$ 'th Snaith map.

Proposition 6.1. The decompositions (7) and (8) coincide.

Proof. We can assume the base is $\operatorname{Spec}(\mathbf{Z})$. We first compute the map

$$
\begin{gathered}
\mathbb{Q}^{\mathbf{Z}} \rightarrow \prod_{j \in \mathbf{Z}} \operatorname{Hom}\left(\Sigma^{2 j, j} \mathrm{LQ}, \Sigma^{2 j, j} \mathrm{LQ}\right) \rightarrow \operatorname{Hom}(\mathrm{PLQ}, \mathrm{PLQ}) \cong \\
\operatorname{Hom}\left(\mathrm{KGL}_{\mathbb{Q}}, \mathrm{KGL}_{\mathbb{Q}}\right) \rightarrow \operatorname{Hom}\left(\Sigma^{-2 i,-i} \Sigma^{\infty} \mathbf{P}_{+}^{\infty}, \mathrm{KGL}_{\mathbb{Q}}\right)=\left(\mathrm{KGL}_{\mathbb{Q}}^{* *} \llbracket x \rrbracket\right)^{2 i, i}=\mathbb{Q} \llbracket u \rrbracket .
\end{gathered}
$$

Here the first map sends a vector $\left(a_{j}\right)_{j \in \mathbf{Z}}$ to the vector $\left(a_{j} \cdot \operatorname{id}_{\Sigma^{2 j, j} \mathbf{L Q}}\right)_{j \in \mathbf{Z}}$. The second map sends a vector of homomorphisms to the sum of the homomorphisms. The third map uses the identification (7). The first map in the second line is precomposition with the $i$ 'th Snaith map. The second identification in the second line uses the identification of the $K G L_{\mathbb{Q}^{-}}$-cohomology of $\mathbf{P}^{\infty}$ with the power series ring over the coefficients induced by the orientation of $\mathrm{KGL}_{\mathbb{Q}}$ coming from the orientation of KGL. The last identification applies an appropriate Bott shift to powers of $x$ to obtain the powers of $u$.

Note that for the system in $i$ on the right hand side all transition maps are the same and given by $u^{j} \mapsto-j u^{j-1}+j u^{j}$ (this follows from the multiplicative formal group law for $\mathrm{KGL}$ ). Note also that we define the Bott element to be the negative reduced class of $\mathbf{P}^{1}$ in $\mathbf{P}^{\infty}$ and BGL.

We let $\operatorname{Hom}\left(\Sigma^{\infty} \mathbf{P}_{+}^{\infty}, \mathbf{P L Q}\right)=\mathbb{Q} \llbracket u^{\prime} \rrbracket$. Here $u^{\prime}$ is the generator for the additive orientation on PLQ times the Bott element.

The multiplicative isomorphism

$$
\mathbb{Q} \llbracket u \rrbracket \cong \operatorname{Hom}\left(\Sigma^{\infty} \mathbf{P}_{+}^{\infty}, \mathrm{KGL}_{\mathbb{Q}}\right) \cong \operatorname{Hom}\left(\Sigma^{\infty} \mathbf{P}_{+}^{\infty}, \mathrm{PLQ}\right) \cong \mathbb{Q} \llbracket u \rrbracket
$$

is given by $u=1-e^{u^{\prime}}$, which is the change of formal parameters between the multiplicative and additive formal group law. 
Moreover the zeroth Snaith map to $\mathrm{KGL}_{\mathbb{Q}}$ is the element

$$
1-u \in \mathbb{Q} \llbracket u \rrbracket \cong \operatorname{Hom}\left(\Sigma^{\infty} \mathbf{P}_{+}^{\infty}, \mathrm{KGL}_{\mathbb{Q}}\right) .
$$

Thus the Snaith map translates to

$$
e^{u^{\prime}} \in \mathbb{Q} \llbracket u^{\prime} \rrbracket \cong \operatorname{Hom}\left(\Sigma^{\infty} \mathbf{P}_{+}^{\infty}, \mathrm{PLQ}\right) \cong \operatorname{Hom}_{\operatorname{Mod}_{\mathbf{S H}(\mathbf{z})}(\mathrm{LQ})}\left(\mathrm{LQ} \wedge \Sigma^{\infty} \mathbf{P}_{+}^{\infty}, \mathrm{PLQ}\right)
$$

We let $\mathbf{L} \mathbf{Q} \wedge \Sigma^{\infty} \mathbf{P}_{+}^{\infty}=\mathbf{L} \mathbf{Q}\left\langle b_{0}, b_{1}, b_{2}, \ldots\right\rangle$, where the $b_{i}$ are dual generators to the powers of the orientation generator. It follows that after these identifications the Snaith map

$$
\mathrm{LQ}\left\langle b_{0}, b_{1}, b_{2}, \ldots\right\rangle \rightarrow \mathrm{PLQ}
$$

has the effect

$$
\mathrm{LQ} b_{j} \stackrel{\frac{1}{j !}}{\rightarrow} \Sigma^{2 j, j} \mathrm{LQ}
$$

on summands.

The Snaith map $\Sigma^{-2 i,-i} \Sigma^{\infty} \mathbf{P}_{+}^{\infty} \rightarrow \mathrm{PLQ}$ is just the same map with the appropriate Bott shift applied and is thus given on summands by

$$
\Sigma^{-2 i,-i} \mathrm{~L} \mathbf{Q} b_{j} \stackrel{\frac{1}{j !}}{\rightarrow} \Sigma^{-2 i+2 j,-i+j} \mathbf{L} \mathbf{Q} .
$$

Now let us start with the $n$ 'th projector $p \in \operatorname{Hom}(\mathrm{PLQ}, \mathrm{PLQ}), n \in \mathbf{Z}$. Composition with the $i$ 'th Snaith map gives us a map

$$
\Sigma^{-2 i,-i} \mathbf{L} \mathbf{Q}\left\langle b_{0}, b_{1}, b_{2}, \ldots\right\rangle \rightarrow \mathrm{PLQ}
$$

sending, if $n \geqslant-i, \Sigma^{-2 i,-i} b_{n+i}$ to $\frac{1}{(2 n+2 i) !} \Sigma^{2 n, n} \mathbf{L Q}$ (abusing notation) and the other generators to 0 . Thus this map is the element

$$
\frac{1}{(2 n+2 i) !} u^{\prime 2 n+2 i} \in \mathbb{Q} \llbracket u^{\prime} \rrbracket=\operatorname{Hom}\left(\Sigma^{-2 i,-i} \Sigma^{\infty} \mathbf{P}_{+}^{\infty}, \text { PLQ }\right) .
$$

Applying the equation $u^{\prime}=\ln (1-u)$ we get the result that the map (9) sends the projector $p$, if $n \geqslant-i$, to

$$
\frac{1}{(2 n+2 i) !} \ln (1-u)^{2 n+2 i} \in \mathbb{Q} \llbracket u \rrbracket=\operatorname{Hom}\left(\Sigma^{-2 i,-i} \Sigma^{\infty} \mathbf{P}_{+}^{\infty}, \mathrm{KGL}_{\mathbb{Q}}\right),
$$

otherwise to 0 .

This is the same formula that is used in $[\mathbf{1 5}]$ to define the projectors, see $[\mathbf{1 5}$, Def. IV.62, after Cor. IV.67, Def. IV.71].

Now it suffices to observe that the Snaith system is indeed the system appearing in [15, Def. IV.62, after Cor. IV.67, Def. IV.71] to describe endomorphisms of KGL and $K \mathrm{KL}_{\mathbb{Q}}$, see $[\mathbf{1 5}$, Th. IV.13, before Prop. IV.33].

Lemma 6.2. The natural map $\mathrm{MGL}_{\mathbb{Q}} \rightarrow \mathrm{LQ}$ factors as an isomorphism $\mathrm{Q}_{\mathbb{Q}} \rightarrow \mathrm{LQ}$.

Proof. The claim follows from the decompositions

$$
\mathrm{MGL}_{\mathbb{Q}} \cong \mathrm{LQ}\left[b_{1}, b_{2}, \ldots\right] \cong \mathbf{L} \mathbf{Q}\left[x_{1}, x_{2}, \ldots\right]
$$

(see [13] for the definition of the $b_{i}$, see also [13, Theorem 10.5]). 
Proposition 6.3. Suppose $S$ is regular. Then the map $\mathrm{MGL}_{\mathbb{Q}} \rightarrow \mathrm{LQ}$ is the map to the zero slice.

Proof. First note that by the decomposition $\mathrm{MGL}_{\mathbb{Q}} \cong \mathbf{L Q}\left[b_{1}, b_{2}, \ldots\right]$ and the effectivity of MGL it follows that $\mathrm{LQ}$ is effective. By lemma (6.2) we have to show that $M G L \rightarrow L Q$ is the map to the zero slice. The decomposition of $M G L_{\mathbb{Q}}$ shows that for this it suffices to show that $\operatorname{Hom}_{\mathbf{S H}(S)}\left(\Sigma^{p, q} \Sigma_{+}^{\infty} X, \mathbf{L Q}\right)=0$ for $X \in \mathrm{Sm} / S$, $p \in \mathbf{Z}, q \geqslant 1$. Equivalently we have to show that every map $\Sigma^{p, q} \Sigma_{+}^{\infty} X \rightarrow \mathrm{KGL}_{\mathbb{Q}}$, $X \in \mathrm{Sm} / S, p \in \mathbf{Z}, q \geqslant 0$, factors through $\mathrm{L} \mathbf{Q}[u]$ with respect to the decomposition $\mathrm{KGL}_{\mathbb{Q}} \cong \mathrm{LQ}\left[u, u^{-1}\right]$. We can assume $q=0$ by replacing $X$ by $X \times \mathbb{G}_{m}^{q}$. Proposition (6.1) and [15, Corollaire VI.75] imply this for non-negative $p$. Suppose $p<0$. Then by the periodicity of PLQ the claim we want to show is equivalent to the statement that for $p+2 q \geqslant 0$ every map $\Sigma^{p+2 q, q} \Sigma_{+}^{\infty} X \rightarrow \mathbf{L} \mathbf{Q}\left[u, u^{-1}\right]$ factors through $u^{q} \mathbf{L} \mathbf{Q}[u]$. This follows from the decomposition [20, Corollary (5.5)(ii)] of the algebraic $K$-theory of the Laurent polynomials over a regular ring.

Corollary 6.4. Suppose $S$ is regular. Then $s_{i}\left(\mathrm{MGL}_{\mathbb{Q}}\right) \cong \Sigma_{T}^{i} \mathrm{~L} \mathbf{Q} \otimes \mathrm{MU}_{2 i}$ compatible with the homomorphism $\mathrm{MU}_{*} \rightarrow \mathrm{MGL}_{\mathbb{Q}, * *}$.

Proof. By proposition (6.3) we have $s_{0}(\mathrm{LQ})=\mathrm{LQ}$. The claim follows from the decomposition $\mathrm{MGL}_{\mathbb{Q}} \cong \mathrm{LQ}\left[x_{1}, x_{2}, \ldots\right]$.

Corollary 6.5. Suppose $S$ is regular. Then $s_{0}\left(\mathbf{1}_{\mathbb{Q}}\right)=s_{0}(\mathbf{1})_{\mathbb{Q}}=\mathrm{LQ}$.

Proof. This follows now from Corollary (3.3).

\section{References}

[1] D.-C. Cisinski and F. Déglise. Triangulated categories of mixed motives, arxiv:0912.2110.

[2] Daniel Dugger and Daniel C. Isaksen. Motivic cell structures. Algebr. Geom. Topol., 5:615-652 (electronic), 2005.

[3] A. D. Elmendorf, I. Kriz, M. A. Mandell, and J. P. May. Rings, modules, and algebras in stable homotopy theory, volume 47 of Mathematical Surveys and Monographs. American Mathematical Society, Providence, RI, 1997.

[4] William Fulton. Intersection theory, volume 2 of Ergebnisse der Mathematik und ihrer Grenzgebiete. 3. Folge. A Series of Modern Surveys in Mathematics [Results in Mathematics and Related Areas. 3rd Series. A Series of Modern Surveys in Mathematics]. Springer-Verlag, Berlin, second edition, 1998.

[5] Philip S. Hirschhorn. Model categories and their localizations, volume 99 of Mathematical Surveys and Monographs. American Mathematical Society, Providence, RI, 2003.

[6] Jens Hornbostel. Localizations in motivic homotopy theory. Math. Proc. Cambridge Phil. Soc., 140(1):95-114, 2006.

[7] Mark Hovey. Model categories, volume 63 of Mathematical Surveys and Monographs. American Mathematical Society, Providence, RI, 1999. 
[8] Thomas Hüttemann. Total cofibres of diagrams of spectra. New York J. Math., 11:333-343 (electronic), 2005.

[9] Tylor Lawson. Motivic homotopy. Notes of a course given by Mike Hopkins and

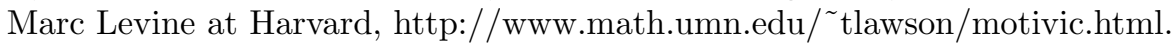

[10] Marc Levine. The homotopy coniveau tower. J. Topol., 1(1):217-267, 2008.

[11] Marc Levine. Comparison of cobordism theories. J. Algebra, 322(9):3291-3317, 2009.

[12] N. Naumann, M. Spitzweck, and P. A. Østvær. Chern classes, $K$-theory and Landweber exactness over nonregular base schemes. In Motives and algebraic cycles, volume 56 of Fields Inst. Commun., pages 307-317. Amer. Math. Soc., Providence, RI, 2009.

[13] N. Naumann, M. Spitzweck, and P. A. Østvær. Motivic Landweber exactness. Doc. Math., 14:551-593, 2009.

[14] Pablo Pelaez. Multiplicative properties of the slice filtration. PhD thesis, arXiv 0806.1704.

[15] Joël Riou. Opérations sur la $K$-théorie algébrique et régulateurs via la théorie homotopique des schémas. Thesis, K-theory Preprint Archives, 793.

[16] M. Spitzweck. Operads, Algebras and Modules in Model Categories and Motives. PhD thesis, University of Bonn, 2001. available at http://hss.ulb.unibonn.de:90/2001/0241/0241.htm.

[17] M. Spitzweck and P. A. Østvær. The Bott inverted infinite projective space is homotopy algebraic K-theory. Bull. Lond. Math. Soc., 41(2):281-292, 2009.

[18] Markus Spitzweck. Slices of motivic Landweber spectra. arXiv:0805.3350v1, to appear in Journal of K-theory.

[19] Markus Spitzweck. Homotopy limits of model categories over inverse index categories. J. Pure Appl. Algebra, 214(6):769-777, 2010.

[20] V. Srinivas. Algebraic $K$-theory, volume 90 of Progress in Mathematics. Birkhäuser Boston Inc., Boston, MA, second edition, 1996.

[21] Gabriele Vezzosi. Brown-Peterson spectra in stable $\mathbb{A}^{1}$-homotopy theory. Rend. Sem. Mat. Univ. Padova, 106:47-64, 2001.

[22] V. Voevodsky. On the zero slice of the sphere spectrum. Tr. Mat. Inst. Steklova, 246(Algebr. Geom. Metody, Svyazi i Prilozh.):106-115, 2004.

[23] Vladimir Voevodsky. Open problems in the motivic stable homotopy theory. I. In Motives, polylogarithms and Hodge theory, Part I (Irvine, CA, 1998), volume 3 of Int. Press Lect. Ser., pages 3-34. Int. Press, Somerville, MA, 2002.

Markus Spitzweck markussp@math.uio.no

Department of Mathematics, University of Oslo, PO Box 1053, Blindern, NO-0316 Oslo, Norway 